\title{
3 Research goals and motivation
}

To our knowledge there is currently no psychometric evaluation tool available for the NIME-community that measures the perceived instrument quality. This work is a first attempt towards the development of a UX-inspired psychometric tool that allows to assess experiential qualities of a musical instrument. These experiential qualities are expected to represent relevant attributes of a musical instrument, and as being perceived as relevant for the musical experience by the musician. The developed questionnaire is expected to allow musicians, designers and researchers to evaluate different facets of the subjectively perceived quality of musical instruments. Because of the intention of exploring its core dimensionality, this work follows an iterative bottom-up approach and is guided by the following goals:

1) Uncover the key experiential qualities of musical instruments from the musician's perspective.

2) Analysis of the importance of those experiential qualities from the perspective of music research experts and the agreement among them.

3) Development of a psychometric measurement tool for the assessment of the musician's perception of experiential qualities of musical instruments. 